\title{
Deep Learning Approaches to Aircraft Maintenance, Repair and Overhaul: A Review
}

\author{
Divish Rengasamy ${ }^{1}$, Hervé P. Morvan ${ }^{1}$, Grazziela P. Figueredo ${ }^{2}$ \\ ${ }^{1}$ Institute for Aerospace Technology, The University of Nottingham, UK \\ ${ }^{2}$ School of Computer Science, The Advanced Data Analysis Centre, The University of Nottingham, UK \\ Email: \{Divish.Rengasamy, Herve.Morvan1, Grazziela.Figueredo\}@ nottingham.ac.uk
}

\begin{abstract}
The use of sensor technology constantly gathering aircrafts' status data has promoted the rapid development of data-driven solutions in aerospace engineering. These methods assist, for instance, with determining appropriate actions for aircraft maintenance, repair and overhaul (MRO). Challenges however are found when dealing with such large amounts of data. Identifying patterns, anomalies and faults disambiguation, with acceptable levels of accuracy and reliability are examples of complex problems in this area. Experiments using deep learning techniques, however, have demonstrated its usefulness in assisting on the analysis aircraft health data. The purpose of this paper therefore is to conduct a survey on deep learning architectures and their application in aircraft MRO. Although deep learning in general is not yet largely exploited for aircraft health, from our search, we identified four main architectures employed to MRO, namely, Deep Autoencoders, Long ShortTerm Memory, Convolutional Neural Networks and Deep Belief Networks. For each architecture, we review their main concepts, the types of problems to which these architectures are employed to, the type of data used and their outcomes. We also discuss how research in this area can be advanced by identifying current research gaps and outlining future research opportunities.
\end{abstract}

\section{INTRODUCTION}

Intelligent Maintenance, Repair and Overhaul (MRO) have become increasingly important in aviation industry. Aircrafts are now fully equipped with sensors that constantly gather information regarding their status, diagnosis and possible faults. The ability to utilise sensor data to accurately predict and diagnose problems facilitates effective maintenance management. In addition, the widespread of sensors in aircrafts has allowed for the transition from time-based maintenance (TBM) activities [1], where maintenance is scheduled under fixed intervals, to condition-based maintenance (CBM), where decisions are based on information collected via sensor monitoring [2], [3]. CBM has enabled the development of both model-based and data-driven methods for aerospace maintenance. Model-based methods are mathematical models of system for fault diagnosis and prognosis [4]. They require domain knowledge to design the models [5]; however, the time necessary to develop a model is higher than that from data-driven methods in aerospace MRO applications [6].

Recently, deep learning approaches have been successfully applied to areas such as audio processing [7], image recognition [8] and self-driving cars [9]. In addition, for several of those examples, deep learning outperforms classical machine learning techniques when tackling large and complex data. Our hypothesis is that these methods will also be successful in big data problems for aircraft MRO, specifically for challenges involving health monitoring [10] and system health management [11]. However, as the area has not been largely exploited and no large datasets have been tested, there are several opportunities and research gaps to be fulfilled to further demonstrate the effectiveness of deep learning in MRO. Our objective with this paper is therefore to study the current approaches and to identify what are their shortcomings, future research, and how our hypothesis can be verified in the future. To the best of our knowledge, there is little literature regarding the survey of deep learning applied to aircraft MRO.

Existing solutions to aircraft MRO problems use four main architectures: Deep Autoencoders (DAE), Long Short-Term Memory (LSTM), Convolutional Neural Networks (CNN) and Deep Belief Networks (DBN). For each architecture, we introduce their main concepts, the problems tackled, the type of data used and their results (Section III). We also identify current research gaps and discuss future research opportunities (Section IIII).

\section{Survey of DeEp Learning Approaches}

In this section we present our survey on deep learning and deep neural network architectures employed in MRO for aircrafts. Although there is not much work yet conducted in the area, from our review we could identify research using four main architectures: DAE, LSTM, CNN and DBN. Table II contains a summary of the current work in the area, as further discussed next.

\section{A. Deep Autoencoders}

DAE [23] is a neural network model that uses a function to map input data into their short/compressed version subsequently decoded into a closest version of the original input. This process forces the encoder to reduce data dimensionality, and in certain cases it learns how to ignore noise. The codification represents the compressed features of input data [24]. DAE therefore has 3 parts: an encoder, a code and a decoder, as shown in Figure 1 DAE encodes the input to the code through hidden layers for a compact representation of the data. Compact representation of data in DAE is used for dimensional reduction. Subsequently, the 
TABLE I

DEEP LEARNING ARCHITECTURES FOR AIRCRAFT PROGNOSIS/DIAGNOSIS

\begin{tabular}{|c|c|c|}
\hline Contribution & Architecture & Application \\
\hline Reddy et al. [12] & Deep autoencoder & $\begin{array}{l}\text { Anomaly detection and fault disambiguation for flyable } \\
\text { electromechanical actuators }\end{array}$ \\
\hline Sarkar et al. [13] & Deep autoencoder & $\begin{array}{l}\text { Crack detection in aircraft's multi-layer composite } \\
\text { sub-elements }\end{array}$ \\
\hline Gao et al. [14] & Deep denoising autoencoder & $\begin{array}{l}\text { remaining useful life prediction in integrated modular } \\
\text { avionics. }\end{array}$ \\
\hline Yuan et al. [15] & Long Short-Term Memory & $\begin{array}{l}\text { Fault diagnosis and remaining useful life estimation of } \\
\text { aero-engine }\end{array}$ \\
\hline ElSaid et al. [16] & Long Short-Term Memory + Ant Colony Optimization [17] & Prognosis of excess vibration in aero-engine \\
\hline Li et al. [18] & Convolutional Neural Network & $\begin{array}{l}\text { Fault diagnosis and remaining useful life estimation of } \\
\text { aero-engine }\end{array}$ \\
\hline Fuan et al. [19] & $\begin{array}{l}\text { Convolutional Neural Network + Particle Swarm } \\
\text { Optimization }\end{array}$ & Rolling bearing fault diagnosis \\
\hline Tamilselvan et al. [20] & Deep Belief Network & Classification of health condition in aero-engines \\
\hline Zhang et al. [21] & Multiobjective Deep Belief Network Ensemble & $\begin{array}{l}\text { Fault diagnosis and remaining useful life estimation of } \\
\text { aero-engine }\end{array}$ \\
\hline Gao et al. [22] & $\begin{array}{l}\text { Deep Belief Networks + Deep Quantum Inspired Neural } \\
\text { Network }\end{array}$ & Fault diagnosis of aircraft's fuel system \\
\hline
\end{tabular}

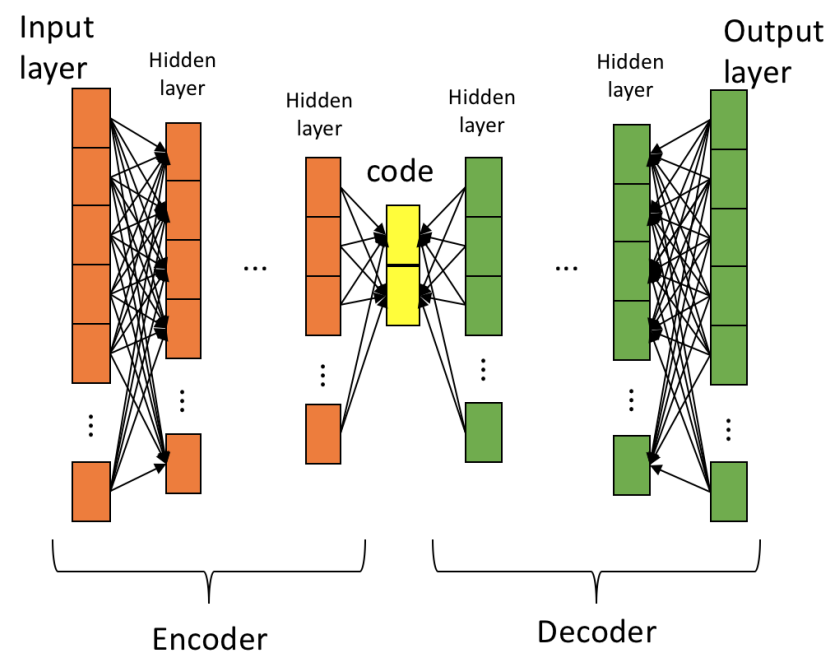

Fig. 1. Structure of a Deep Autoencoder

output is reconstructed from the code through decoder for anomaly detection or generating new data.

For Aerospace MRO, DAEs are employed for structural health monitoring and anomaly detection. Sarkar et al. [13], for instance, uses DAE for Structural Health Monitoring - specifically for detection of cracks in thick multi-layer composites in aircrafts. The authors analyse videos of the composite coupons slowly bent until full fracture. Those video frames containing images of composite coupon without cracks are used to train the DAE. Frames of images with cracks are used as test. The crack detection is based on the magnitude of reconstruction error between the input and output images. The authors obtained good results for crack detection using DAE; however, the weaknesses of their approach were not assessed, as there is no performance comparison with alternative methods.

Reddy et al. [12] employs DAE on aircraft data for anomaly detection and fault disambiguation. Time series data from multiple sensors (without faults) are first normalised to zero mean and unit variance. Subsequently, DAE employs a subsection of the data with a fixed time window as input. The DAE training considers around 100,000 data points using the Backpropagation algorithm with gradient descent to reconstruct the inputs. The fault detection occurs based on the reconstruction error between the input and the output using the Root Mean Square (RMS) error as a metric for disparity. When a fault is present in the data, the reconstruction error is large because the DAE is trained to output data without faults. The data set employed by the authors is obtained from an experiment for fault scenarios, under various operating conditions of Flyable Electromechanical Actuator (FLEA) performed by Balaban et al. in [25] and [26]. The data set contains 13 dimensions (time, actuator $\mathrm{Z}$ position, measured load, motor $\mathrm{X}$ current, motor $\mathrm{Y}$ current, motor $\mathrm{Z}$ current, motor $\mathrm{X}$ voltage, motor $\mathrm{Y}$ voltage, motor $\mathrm{Y}$ temperature, motor $\mathrm{Z}$ temperature, nut $\mathrm{X}$ temperature, nut $\mathrm{Y}$ temperature, ambient temperature), which are sampled at $100 \mathrm{~Hz}$. The data employed for testing the DAE have a total of 95 nominal runs and 255 faulty runs, with each run containing a window of approximately $30 \mathrm{~s}$ of data. The faulty runs contain examples of both spall and ballscrew jam faults. Using an 11-layer DAE and a 14-dimensional bottleneck layer, the trained DAE is able to achieve a fault detection rate of $97.8 \%$ and $0.0 \%$ of false positives. In addition, DAE is able to cluster the different fault types based on the distribution of reconstruction error over multiple sensors.

Gao et al. [14] utilizes a combination of stacked denoising autoencoders [27] (SDAE) and Support Vector Machine (SVM) to predict the Remaining Useful Life (RUL) of 
integrated modular avionics (IMA). Degradation of IMA is typically caused by the wearing out of electronics that lead to electromigration and time-dependent dielectric breakdown - the root cause of intermittent faults (IF) in IMA. IMA is a network of multiple computing modules used in aircraft with various functionalities of different critical levels. Therefore, identifying faults and predicting the RUL of IMA are crucial for maintaining the integrity of the aircraft. A simulation of the IMA including IF and degradation is performed using Simulink to collect the data. The simulation consists of 12000 fly cycles and IF are injected using the Monte Carlo method. Subsequently, a SDAE is used to extract the most salient features from the raw data. The structure of the SDAE consists of 5 hidden layers with 48, 20 1, 20 and 48 nodes for each hidden layer. In addition, the SDAE is pretrain and fine-tune with 800 and 400 epochs respectively. Next, the extract features are employed by SVM to construct a model to predict the RUL. Finally, the results achieved by SDAE with SVM outperform the conventional method by a wide margin. However, the authors did not provide the model used in the conventional method.

\section{B. Long Short-Term Memory}

LSTM [28] is a type of recurrent neural network designed with chain units consisting of input, forget and output gates. Each gate is wrapped with a sigmoid function. The input gate controls the influence of the current input. The forget gate within each unit controls how much information needs to be retained. The output gate decides whether the flow will be passed on to the next LSTM unit. This architecture allows for the learning of long-term dependencies of data. LSTM is currently employed in the aerospace MRO for fault diagnosis, remaining useful life estimation and prognosis of excess vibration in aero-engines.

ElSaid et al. [16], for example, uses a combination of LSTM and Ant-Colony Optimization [17] (ACO) to predict the occurrence of excess vibration in aero-engines. Domain expertise assisted with selecting 15 features amongst 72 for their model. The authors compare 3 LSTM architectures: (i) 10 time steps (current time unit +9 previous time units) for each feature and 2 hidden layers; (ii) 10 time steps and 1 hidden layer; and (iii) 20 time steps and 3 hidden layers. The LSTM architectures are required to predict the magnitude of the aero-engine vibration within 1, 5, 10 and 20 seconds. The authors conclude that all architectures achieve good results. Further improvement is achieved by LSTM optimisation via ACO.

Yuan et al. [15] uses LSTM to predict RUL and to classify faults of aircraft turbofan engines. A LSTM with 3 inputs and 2 outputs is considered. The inputs are the sample number, the time-step and the engine's sensor value. The outputs are the RUL estimation and the fault diagnosis. A static hidden layer is added to improve fault classification. The cost function is the Mean Squared Error (MSE). Furthermore, the authors use dropout and early stopping mechanisms to increase generalisation and prevent overfitting. The Adam [29] optimiser is employed to the gradient descent [30]. The same optimisation process is applied to a standard RNN, to a Gated Recurrent Unit (GRU) [31] and to an AdaBoost LSTM model for results comparison. Aircraft gas turbine engines and its damage propagation are simulated using the Commercial Modular Aero-Propulsion System Simulation (C-MAPSS), developed by NASA. The data obtained from the the simulation contains 21 engine variables, including normal, fault onset and system failure operations. Among the fault onset and system failure data, there are four different classes: (i) high pressure compressor failure with single operating condition in engines; (ii) high pressure compressor failure with six operating conditions in engines; (iii) high pressure compressor and fan degradation of engines with a single operating condition; (iv) high pressure compressor and fan degradation of engines with six operating conditions. Faults are inserted at random times for each simulation. The engine health is indicated by the efficiency and flow of its components. When either efficiency or flow reaches zero, the simulated engine has failed. A SVM is employed to label the fault category for each time step. All labels prior to fault have the maximum RUL value; and after the fault, RUL value decreases according to a power function. Input data are normalised using mean and variance; output is normalised by dividing the RUL by the minimum lifespan of aero-engine within the data set. The RUL estimation is evaluated using a relative error between the actual and estimated error that are less than or equal to $5 \%, 10 \%$ and $20 \%$ respectively. In addition a score based on the standard asymmetric scoring function, proposed by Saxena et al. in [32] is considered. Results reveal that the LSTM outperforms all other methods investigated for both RUL estimation and fault occurrence predictions.

\section{Convolutional Neural Networks}

CNN [33] are neural networks that contain convolution layers with nonlinear activation functions and a fully connected layer at the end to compute the outputs. The input data goes through each convolution layer with different filters and is combined in the end for a result. This allows CNN to perform better on data that has high spatial correlation with its neighbourhood data-points. Figure 2 shows how the spatial relationship within data are preserve through convolution using a Kernel.

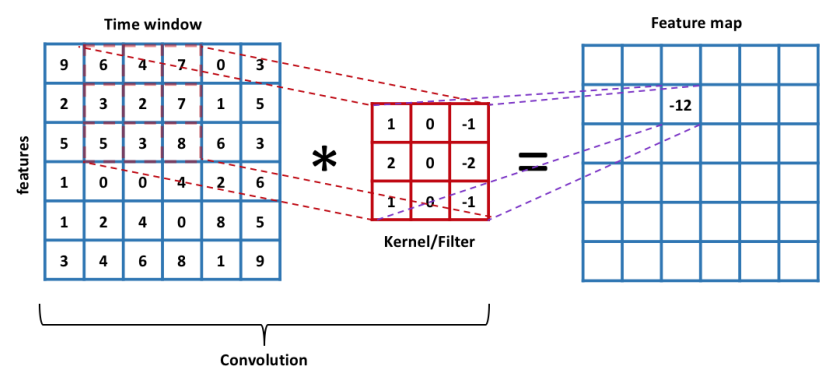

Fig. 2. Example of convolution operation on 2D data. Input data and Kernel are convolved to produce a feature map. 
In aerospace MRO, CNN is currently used for rolling bearing fault diganosis, RUL estimation of aero-engines and fault diagnosis in aero-engine.

Fuan et al. [19] employs adaptive deep CNN and Particle Swarm Optimisation (PSO) [34] to diagnose and classify fault in rolling bearing. PSO is employed to optimise the parameters of the CNN. Experimental data are acquired from an electrical locomotive rolling bearing test rig. The data collected from test rig are vibration signals with 8 different health condition that includes 1 normal condition and 7 faulty conditions. Subsequently, 4 different methods: (i) CNN with PSO; (ii) CNN; (iii) SVM; and (iv) Artificial Neural Networks (ANN) Multilayer Perceptron are compared using both raw and pre-processed data. One of the main advantages of $\mathrm{CNN}$ is that it does not require manual feature extraction. The convolution layers automatically extract relevant features, which are used to diagnose faults in rolling bearing. Results comparison is based on the percentage of correct classification. In both experiments, $\mathrm{CNN}+\mathrm{PSO}$ achieved the highest classification accuracy. Moreover, the experiment shows that better results are achieved with pre-processed data.

Li et al. [18] uses deep CNN to estimate RUL and fault diagnosis of aircraft turbofan engines. The authors employ a conventional $\mathrm{CNN}$ with 4 convolution layers for feature extraction and a fully-connected layer for regression. The network requires 2 inputs, comprising of the time sequence of data and selected features. A 1-dimensional kernel filter is applied to each convolution layer. The size of the kernel filter corresponds to the data that represent local features. A medium size and number kernel filter is chosen to balance accuracy and computational cost. Furthermore, zero-padding is added to each convolution layer to maintain the dimension of the feature map. Subsequently, the feature map is flattened into a one-dimensional feature vector to be added to the full-connected layer for regression. The dropout technique is used to prevent overfitting. In addition, the Xavier normal initialiser [35] determines the initial weights, while the Adam optimiser is employed for gradient descent. The data set is normalised to $[-1,1]$ and given the RUL labels. Training is conducted using mini-batch gradient descent [30]. Similarly to Yuan et al. discussed in Section II-B, RUL is evaluated using the asymmetric scoring function [32] and RMSE. Results from the CNN are compared to other methods such as LSTM, RULCLIPPER [36], Random Forest, Gradient Boosting, SVM, Echo State Network with Kalman filter [37], Multi-objective deep belief networks ensemble [21] and Time window-based NN [38]. C-MAPSS data is employed for testing. Results reveal that CNN outperforms LSTM, RNN, Deep Neural Network (DNN) for RMSE. CNN also achieved the lowest RMSE. The authors also show that training time increases proportionally to the number of convolution layers and conclude that the optimal number layers is 5 for their problem.

\section{Deep Belief Networks}

DBN [39] is employed in aerospace MRO for applications such as aero-engine fault classification, aero-engine RUL estimation and aircraft fuel system fault diagnosis. The DBN is a generative graphical model with stacked Restricted Boltzmann Machine (RBM) [40]. Each RBM composes of a hidden and visible layer with connections between layers but not within each layer. DBN is trained in a greedy layerwise unsupervised manner to extract features from the input data. Tamilselvan et al. [20] uses a DBN classifier to identify the health state of aero-engine. C-MAPSS data is employed. The DBN classifier used consists of 3 hidden-layers. Conjugate gradient approach [39] are used to fine-tune the DBN classifier after it has been pre-trained and trained. DBN fault classification of aero-engines is compared to SVM, Backpropagation Neural Network (BNN), Self-Organizing Maps [41] and Mahalanobis Distance. Results shows that DBN achieves the best fault classification accuracy for 5 of the 6 operating conditions.

Zhang et al. [21] uses Multiobjective Deep Belief Networks Ensemble (MODBNE) to estimate RUL of aeroengines using the C-MAPSS data. Each DBN parameter is generated by a Multiobjective Evolutionary Algorithm based on Decomposition (MOEA/D). The parameters evolved are (i) the number of hidden neurons per hidden layer; (ii) the weight cost of contrastive divergence; (iii) the learning rates to update weights and biases for backpropagation. Contrastive divergence and backpropagation are employed to train the DBNs. A population of 20 candidate solutions for DBNs and their parameters are randomly initialised. The trained DBNs are subject to optmization of two conflicting objectives. The two objectives are minimisation of the DBNs prediction error and maximisation of the diversity of outputs between DBNs. High diversity among DBNs leads to better generalisation performance of the ensemble [42]. Subsequently, MOEA/D is used to evolve the parameters of DBNs; these new parameters are use to re-train the DBNs. The optimised DBNs are combined using single-objective differential evolution to create the ensemble. Results show that MODBNE achieves the most accurate estimation of RUL when compared to 10 other data-driven methods. The authors also show that 3 hidden layers and time window size of 30 achieve the best performance.

One of the most important components in an aircraft is the fuel system, as it controls the fuel flow to the engine. Failure or fault in fuel system causes instability to the engine and in the auxiliary power unit. Gao et al. [22] uses Deep Quantum Inspired Neural Network (DQINN), a method inspired by Deep Quantum Network (DQN) [43] for aircraft fuel system fault diagnosis. DQINN is a combination of DBN and Quantum Inspired Neural Network (QINN) [44]. QINN differs from traditional NN on its activation functions. QINN have multilevel activation functions to assist in fuzzy classification. To simulate faults in the aircraft fuel system system, 4 common faults are added into the system: (i) motor phase power turned off; (ii) rise in resistance of 
motor phase stator; (iii) increase of fluid loss in vanes; and (iv) increase in leakage of boost pump. After the faults have been injected into the model, the pressure at the input of the engine is recorded. Subsequently, DQINN uses the data to form a fault classifier. In the initialisation step, the DBN are pre-trained using unsupervised learning. This allows for better generalisation of the training data set [45]. The output from DBN are the input for QINN. Each level of multilevel activation function in QINN has a unique quantum interval and each level require different parameters for DBN. Therefore, DBN are duplicated at each level of activation function in QINN to allow DBN parameters to be updated accordingly. Using the MSE as the accuracy metric, DQINN's performance is compared to DQN, Classical Deep Belief Network (CDBN) and BNN. The sampling frequency of the aircraft fuel system's Simulink model to obtain the data is $8 \mathrm{kHz}$ and all 4 failure mode are simulated in $0.125 \mathrm{~s}$ resulting in a total 1000 data points split equally among 4 faults. Subsequently, features are extracted from the raw data based on mean, root mean square, skewness and Kurtosis, reducing the data to 400 points. Results reveal that DQINN achieves the highest fault classification accuracy. DQINN also achieves the lowest standard deviation compared to DQN, CDBN and BNN.

\section{Future Opportunities}

Deep learning has enabled new research opportunities and the development of novel tools to assist in aircraft's MRO activities. Our survey has revealed that the use of deep neural networks leads to successful outcomes in this area. There are however many possibilities for improvement, which have not yet been investigated. In this section, we outline and discuss some of these opportunities that we believe are relevant to advance MRO research.

\section{A. Alternative Deep Learning architectures}

The previous section shows that for aerospace, currently there is a small subset of deep neural networks architectures employed to MRO activities. However, in the reviewed work the rationale for the choice of deep learning as well as a specific architecture is often unclear. Specific problems in aerospace MRO require specialist deep neural network architectures to achieve desirable results. A large knowledge of different deep neural network architectures can reduce time required to select the most suitable architecture. In Table III. a list of popular deep learning architectures along with its pros and cons are summarised to assist in the selection of appropriate deep neural networks architecture.

\section{B. Tackling Uncertainty}

For safety-critical applications, it is important to know the confidence level of the deep neural networks output. This confidence level depends on the epistemic and aleatory uncertainty inherent in the data set investigated. The epistemic uncertainty refers to uncertainty in the model due to limited data and knowledge - that is, the data the model has never been exposed to. On the other hand, aleatory uncertainty regards the natural randomness within a system that cannot be explained with more data. Both types of uncertainty can propagate through the deep learning model, affecting its outcome. One alternative to overcome this issue is by employing Bayesian approaches [48] or fuzzy methods [49] to tackle uncertainty. In addition to quantifying the uncertainty of the model's prediction, the ability to explain and interpret the predictions are often desirable. Greater interpretability in prediction can be achieved by employing the method such as Local Interpretable Model-Agnostic Explanations [50].

\section{Active Learning for Data Labelling}

Aircraft generate high volume of sensor data during each flight. These data however are typically unlabelled; and the training process of a supervised learning model requires a large number of labelled instances. Labelling these data sets manually is almost unfeasible, due to its cost and time consumption. A possible strategy to overcome expensive manual labelling is the application of Active Learning [51] for this task. In this approach, algorithm such as queryby-committee [52] determine the data point that optimises learning and actively request data label from domain experts. Thus, this allows active learning algorithms to learn from substantially less data.

\section{The Creation of a Benchmark Data Repository}

A major hindrance for researchers in the aircraft MRO area is the lack of benchmark/standard big data sets to evaluate MRO solution approaches. Currently, C-MAPSS appears to be the standard test data set to compare different deep neural networks architectures for aero-engine MRO problems. However, this data set alone is not sufficient to attest the effectiveness of the deep learning approach. In addition, other parts and components of the aircraft monitored by sensors are not yet being considered due to the lack of test benchmarks. Therefore, a publicly available data repository for various components of an aircraft would assist for better testing and comparison of approaches, therefore advancing the research in this area.

\section{Conclusions}

The increased usage of sensors for data collection in aircrafts has created the need for sophisticated solutions to interpret the large volumes of data. In particular, for aerospace engineering, these solutions are important for MRO applications.

Although not yet largely exploited, deep learning achieved relative success for MRO problems. In this paper we have performed a survey of the current deep learning approaches and applications to MRO. From our review we observed that deep learning models often outperform model-based and statistical machine learning techniques. Architectures such as DAEs, LSTMs, CNNs, DBNs and some of their hybrid forms are currently being employed for diagnosis, anomaly detection and prognosis in parts of the aircraft, such as aeroengines, fuel systems and actuators. 
TABLE II

ALTERNATIVE DEEP LEARNING ARCHITECTURES: OPPORTUNITIES FOR AIRCRAFT MAINTENANCE, REPAIR AND OVERHAUL ACTIVITIES.

\begin{tabular}{|c|c|c|c|}
\hline Architectures & Descriptions & Advantages & Disadvantages \\
\hline Variational autoencoder [46] & $\begin{array}{l}\text { A generative version of } \\
\text { autoencoder. It learns the original } \\
\text { data distribution and generates new } \\
\text { data with similar distribution. }\end{array}$ & $\begin{array}{l}\text { It learns hidden representation and } \\
\text { generates new data }\end{array}$ & $\begin{array}{l}\text { Difficult to implement and optimise } \\
\text { compared to other variants of } \\
\text { autoencoder. }\end{array}$ \\
\hline Contractive autoencoder [47] & $\begin{array}{l}\text { Penalises autoencoder's sensitivity } \\
\text { to training input. }\end{array}$ & $\begin{array}{l}\text { Higher robustness and better } \\
\text { generalisation }\end{array}$ & $\begin{array}{l}\text { Computation of Jacobian hidden } \\
\text { layer causes higher complexity } \\
\text { compared to denoising autoencoder. }\end{array}$ \\
\hline Recurrent Neural Network (RNN) & $\begin{array}{l}\text { It has an internal state and memory } \\
\text { to process sequence or temporal } \\
\text { data }\end{array}$ & $\begin{array}{l}\text { Easy implementation and suitable } \\
\text { for sequential data }\end{array}$ & $\begin{array}{l}\text { Problems with connecting } \\
\text { long-term dependencies in data. }\end{array}$ \\
\hline Gated Recurrent Unit (GRU) & $\begin{array}{l}\text { A variant of LSTM with simplified } \\
\text { hidden units and fewer parameters }\end{array}$ & $\begin{array}{l}\text { Training occurs faster due to its } \\
\text { low complexity }\end{array}$ & $\begin{array}{l}\text { Compared to RNNs, however, more } \\
\text { difficult to train and implement due } \\
\text { to higher complexity. }\end{array}$ \\
\hline
\end{tabular}

There are however, several gaps and research opportunities yet to be pursued. Several alternative deep learning architectures have not yet been tested for MRO problems. In particular, there is little literature on the application of methods such as Deep Bayesian Neural Networks and hybridisations with fuzzy logic. Active learning for targeted labelling of data is also another avenue to facilitate classification. Finally, we draw the attention to the fact that no large data has yet been employed to confirm that deep learning outperforms other intelligent MRO methods. The creation of a repository of benchmark big data sets, where researchers could test and compare their machine learning approaches for aerospace MRO is therefore necessary.

\section{ACKNOWLEDGMENT}

This work is funded by the INNOVATIVE doctoral programme. The INNOVATIVE programme is partially funded by the Marie Curie Initial Training Networks (ITN) action (project number 665468) and partially by the Institute for Aerospace Technology (IAT) at the University of Nottingham.

\section{REFERENCES}

[1] G. P. Figueredo, K. Owa, and R. I. John, "Multi-Objective Optimization for Preventive Maintenance in Transportation: A Review," University of Nottingham, Tech. Rep., 2018.

[2] R. Ahmad and S. Kamaruddin, "An overview of time-based and condition-based maintenance in industrial application," Computers and Industrial Engineering, vol. 63, no. 1, pp. 135 - 149, 2012.

[3] J. Kim, Y. Ahn, and H. Yeo, "A comparative study of time-based maintenance and condition-based maintenance for optimal choice of maintenance policy," Structure and Infrastructure Engineering, vol. 12, no. 12, pp. 1525-1536, 2016.

[4] J. Gertler, "Survey of model-based failure detection and isolation in complex plants," IEEE Control Systems Magazine, vol. 8, 1988.

[5] S.-J. Kim and C.-W. Lee, "Diagnosis of sensor faults in active magnetic bearing system equipped with built-in force transducers," IEEE/ASME Transactions on Mechatronics, vol. 4, 1999.

[6] P. Freeman et al., "Model-based and data-driven fault detection performance for a small uav," IEEE/ASME Transactions on Mechatronics, vol. 18, 082013.

[7] H. Lee et al., "Unsupervised feature learning for audio classification using convolutional deep belief networks," in Proc of NIPS'09, 2009, pp. 1096-1104.
[8] K. Simonyan and A. Zisserman, "Very deep convolutional networks for large-scale image recognition," International Conference on Learning Representations (ICLR), vol. abs/1409.1556, 2014.

[9] M. Bojarski et. al, "End to end learning for self-driving cars," CoRR, vol. abs/1604.07316, 2016.

[10] R. Zhao et al., "Deep learning and its applications to machine health monitoring: A survey," CoRR, vol. abs/1612.07640, 2016.

[11] S. Khan and T. Yairi, "A review on the application of deep learning in system health management," Mec Sys and Sig Proc, vol. 107, 2018.

[12] K. K. Reddy, S. Sarkar, V. Venugopalan, and M. Giering, "Anomaly Detection and Fault Disambiguation in Large Flight Data: A Multimodal Deep Auto-encoder Approach," Prognostics and Health Management, no. i, pp. 1-8, 2016.

[13] S. Sarkar et al., "Deep learning for structural health monitoring: A damage characterization application," An Conf of The Prog and Health Mangmt Soc, 2016.

[14] Z. Gao, C. Ma, and Y. Luo, "Rul prediction for ima based on deep regression method," in 2017 IEEE 10th International Workshop on Computational Intelligence and Applications (IWCIA), Nov 2017, pp. 25-31.

[15] M. Yuan et al., "Fault diagnosis and remaining useful life estimation of aero engine using LSTM neural network," IEEE Int Conf on Aircraft Utility Systems, pp. 135-140, 102016.

[16] A. ElSaid et. al, "Optimizing LSTM RNNs using ACO to predict turbine engine vibration," in Proc of the GECCO'2017, 2017, pp. 2122.

[17] M. Dorigo and T. Stützle, Ant Colony Optimization. Scituate, MA, USA: Bradford Company, 2004.

[18] X. Li et al., "Remaining useful life estimation in prognostics using deep convolution neural networks," Rel Eng \& Sys Safety, vol. 172, 122017.

[19] W. Fuan et al., "An adaptive deep convolutional neural network for rolling bearing fault diagnosis," Meas Sci and Tech, vol. 28, no. 9, 2017.

[20] P. Tamilselvan and P. Wang, "Failure diagnosis using deep belief learning based health state classification," Rel Eng \& Sys Safety, vol. 115, 2013.

[21] C. Zhang et al., "Multiobjective deep belief networks ensemble for remaining useful life estimation in prognostics," IEEE Tran on Neural Nets and Learning Systems, vol. PP, pp. 1-13, 072016.

[22] Z. Gao, C. Ma, D. Song, and Y. Liu, "Deep quantum inspired neural network with application to aircraft fuel system fault diagnosis," Neurocomput., vol. 238, no. C, pp. 13-23, May 2017.

[23] G. Hinton and R. Salakhutdinov, "Reducing the dimensionality of data with neural networks," vol. 313, pp. 504-7, 082006.

[24] I. Goodfellow, Y. Bengio, and A. Courville, Deep Learning. MIT Press, 2016.

[25] E. Balaban et al., "Modeling, detection, and disambiguation of sensor faults for aerospace applications," Sensors J., vol. 9, 2009.

[26] — - "Prognostic health-management system development for electromechanical actuators," J. of Aerosp Inf Sys, vol. 12, 2015.

[27] P. Vincent, H. Larochelle, Y. Bengio, and P.-A. Manzagol, "Extracting 
and composing robust features with denoising autoencoders," in ICML, 2008, pp. 1096-1103.

[28] S. Hochreiter and J. Schmidhuber, "Long short-term memory," Neural Comput., vol. 9, no. 8, pp. 1735-1780, Nov. 1997.

[29] D. P. Kingma and J. Ba, "Adam: A method for stochastic optimization," CoRR, vol. abs/1412.6980, 2014.

[30] S. Ruder, "An overview of gradient descent optimization algorithms," CoRR, vol. abs/1609.04747, 2016.

[31] K. Cho et al., "Learning phrase representations using RNN encoderdecoder for statistical machine translation," CoRR, vol. abs/1406.1078, 2014.

[32] A. Saxena et al., "Damage propagation modeling for aircraft engine run-to-failure simulation," in I Conf on Prog and Health Man, Oct 2008.

[33] Y. LeCun et al., "Backpropagation applied to handwritten zip code recognition," Neural Comp, vol. 1, no. 4, pp. 541-551, 1989.

[34] J. Kennedy and R. C. Eberhart, "Particle swarm optimization," in IEEE I Conf on Neural Networks, 1995, pp. 1942-1948.

[35] X. Glorot and Y. Bengio, "Understanding the difficulty of training deep feedforward neural networks," in I Conf on Art Int and Stats, 2010.

[36] E. Ramasso, "Investigating computational geometry for failure prognostics," International Journal of Prognostics and Health Management (2153-2648), vol. 005, pp. 1-18, 072014.

[37] Y. et al. Peng, "A modified echo state network based remaining useful life estimation approach," in IEEE C. on Prog and Health Man, 2012.

[38] L. Pin, C.-K. Goh, and K. Chen Tan, "A time window neural network based framework for remaining useful life estimation," in Int Joint Conf on Neural Networks, 07 2016, pp. 1746-1753.

[39] G. E. Hinton, S. Osindero, and Y. W. Teh, "A fast learning algorithm for deep belief nets," Neural Comp, vol. 18, no. 7, pp. 1527-1554, 2006.

[40] R. Salakhutdinov et al., "Restricted boltzmann machines for collaborative filtering," in 24th Int Conf on Machine Learning, 2007, pp. 791-798.

[41] T. Kohonen, "The self-organizing map," Proceedings of the IEEE, vol. 78, no. 9, pp. 1464-1480, Sep 1990.

[42] G. Brown, J. Wyatt, R. Harris, and X. Yao, "Diversity creation methods: a survey and categorisation," Inf Fusion, vol. 6, no. 1, pp. 5 $-20,2005$.

[43] S. Zhou, Q. Chen, and X. Wang, "Deep quantum networks for classification," Pattern Recognition(ICPR), pp. 2885 - 2888, 092010.

[44] T. Menneer and A. Narayanan, "Quantum-inspired neural networks," Tech. Rep., 1995.

[45] D. Erhan et al., "Why does unsupervised pre-training help deep learning?" J. Mach. Learn. Res., vol. 11, pp. 625-660, 2010.

[46] D. P. Kingma and M. Welling, "Auto-encoding variational bayes," CoRR, vol. abs/1312.6114, 2013.

[47] S. Rifai et al., "Contractive auto-encoders: Explicit invariance during feature extraction," in 28th ICML, 2011, pp. 833-840.

[48] M. A. Zaidan, A. R. Mills, and R. F. Harrison, "Bayesian framework for aerospace gas turbine engine prognostics," in 2013 IEEE Aerospace Conference, 2013.

[49] C. Chen, B. Zhang, and G. Vachtsevanos, "Prediction of machine health condition using neuro-fuzzy and bayesian algorithms," vol. 61, pp. 297-306, 022012

[50] M. T. Ribeiro, S. Singh, and C. Guestrin, "Why should i trust you?": Explaining the predictions of any classifier," in Proceedings of the 22Nd ACM SIGKDD International Conference on Knowledge Discovery and Data Mining, ser. KDD '16, 2016, pp. 1135-1144.

[51] B. Settles, "Active learning literature survey," University of Wisconsin-Madison, Computer Sciences Technical Report 1648, 2009.

[52] H. S. Seung, M. Opper, and H. Sompolinsky, "Query by committee," in Fifth A. Work. on Comp Learning Theory, 1992, pp. 287-294. 